\title{
Quantitative spectroscopic analyses in the IACOB+OWN project: Massive O-type stars in the Galaxy with the current Gaia information
}

\section{Gonzalo Holgado, ${ }^{1,2}$, Sergio Simón-Díaz ${ }^{1,2}$ and Rodolfo Barbá ${ }^{3}$}

${ }^{1}$ Instituto de Astrofísica de Canarias, E-38200 La Laguna, Tenerife, Spain.

${ }^{2}$ Departamento de Astrofísica, Universidad de La Laguna, E-38205 La Laguna, Tenerife, Spain.

${ }^{3}$ Departamento de Física y Astronomía, Univ. de la Serena, Av. Juan Cisternas 1200 Norte, La Serena, Chile

We present the results from the quantitative spectroscopic analysis of $\sim 280$ likely single $\mathrm{O}$ stars targeted by the IACOB and OWN surveys. This implies the largest sample of Galactic O-type stars analyzed homogeneously to date. We used the IACOB-BROAD and IACOB-GBAT tools (see Simón-Díaz et al. 2011,2015) to obtain the complete set of spectroscopic parameters which can be determined from the optical spectrum of O-type stars: projected rotational velocity $(v \sin i)$, macroturbulence velocity $\left(v_{\text {mac }}\right)$, effective temperature $\left(T_{\text {eff }}\right)$, gravity $(\log g)$, wind-strength $(\log Q)$, helium abundance $\left(Y_{\mathrm{He}}\right)$, microturbulence $\left(\xi_{\mathrm{t}}\right)$, and the exponent of the wind-law $(\beta)$.

The results presented in this poster contribution can be also found in Holgado et al.(2016). These include a summary of the present completitude in magnitude of our studied sample, the distribution of projected rotational velocities and an overview of the distribution of the stellar properties in the spectroscopic HR diagram. We also evaluate the present situation regarding available information about distances, as provided by the Hipparcos and Gaia missions. We find that the parallaxes in TGAS-DR1 are still not good enough to derive accurate radii, luminosities and masses, as only $2 \%$ of our sample have parallaxes with an uncertainty lower than $20 \%$.

For the next steps we plan to :

- Investigate the distribution of projected rotational velocities in Galactic O stars.

- Revisit calibrations of stellar parameters with spectral type and luminosity class.

- Study the origin of the absence of stars near the ZAMS in the range 20-60 M $\odot$.

- Perform the abundance analysis of the sample.

And, once Gaia will achieve more reliable values of distances (as already expected for DR2):

- Provide a homogeneous and statistically significant empirical overview of the physical properties of Galactic O-type stars.

- Use these results to constraint theoretical predictions about the initial phases of massive stars evolution and evaluate the theory of radiatively-driven winds in the Galactic O Stars.

\section{References}

Simón-Díaz, S., Castro, N., Garcia, M., Herrero, A., \& Markova, N. 2011d, Bulletin de la Societe Royale des Sciences de Liege, 80, 514

Simón-Díaz, S., Negueruela, I., Maíz Apellániz, J., et al. 2015, Highlights of Spanish Astrophysics VIII, 576

Holgado, G., Simón-Díaz, S., \& Barbá, R. H. 2016, arXiv:1611.02634 